\title{
The assisted bidirectional Glenn: A novel surgical approach for first-stage single-ventricle heart palliation
}

\author{
Mahdi Esmaily-Moghadam, PhD, ${ }^{a}$ Tain-Yen Hsia, MD, ${ }^{b}$ and Alison L. Marsden, $\mathrm{PhD},{ }^{\mathrm{a}}$ for the Modeling of \\ Congenital Hearts Alliance (MOCHA) Investigators
}

\begin{abstract}
Background: Outcomes after a modified Blalock-Taussig shunt (mBTS) in neonates with single-ventricle physiology remain unsatisfactory. However, initial palliation with a superior cavopulmonary connection, such as a bidirectional Glenn (BDG), is discouraged, owing to potential for inadequate pulmonary blood flow (PBF). We tested the feasibility of a novel surgical approach, adopting the engineering concept of an ejector pump, whereby the flow in the BDG is "assisted" by injection of a high-energy flow stream from the systemic circulation.

Methods: Realistic 3-dimensional models of the neonatal mBTS and BDG circulations were created. The "assisted" bidirectional Glenn (ABG) consisted of a shunt between the right innominate artery and the superior vena cava (SVC), with a 1.5-mm clip near the SVC anastomosis to create a Venturi effect. The 3 models were coupled to a validated hydraulic circulation model, and 2 pulmonary vascular resistance (PVR) values (7 and 2.3 Wood units) were simulated.
\end{abstract}

Results: The ABG provided the highest systemic oxygen saturation and oxygen delivery at both PVR levels. In addition to achieving higher PBF than the BDG, the ABG produced a lower single-ventricular workload than mBTS. SVC pressure was highest in the ABG model (ABG: 15; Glenn: 11; mBTS: $3 \mathrm{~mm} \mathrm{Hg}$; PVR $=7$ Wood units), but at low PVR, the SVC pressure was significantly lower (ABG: 8; Glenn: 6; mBTS: $<3 \mathrm{~mm} \mathrm{Hg}$ ).

Conclusions: Adopting the principle of an ejector pump, with additional flow directed into the SVC in a BDG, the ABG appears to increase PBF with a modest increase in SVC and pulmonary arterial pressure. Although multiscale modeling results demonstrate the conceptual feasibility of the ABG circulation, further technical refinement and investigations are necessary, especially in an appropriate animal model. (J Thorac Cardiovasc Surg 2015;149:699-705)

See related commentary on pages 706-7.

From the Mechanical and Aerospace Engineering Department, ${ }^{\mathrm{a}}$ University of California, San Diego, La Jolla, Calif; and Great Ormond Street Hospital for Children and University College London Institute of Cardiovascular Science, London, United Kingdom.

This work was supported by the Leducq Foundation as part of the Transatlantic Network of Excellence for Cardiovascular Research, a Burroughs Wellcome Fund Career Award at the Scientific Interface, and National Science Foundation CAREER OCI-1150184. The authors acknowledge the open-source Simvascular project (simtk.org).

Disclosures: Authors have nothing to disclose with regard to commercial support. MOCHA Investigators: Andrew Taylor, MD; Alessandro Giardini, MD; Sachin Khambadkone, MD; Silvia Schievano, PhD; Marc de Leval, MD; and T.-Y. Hsia, MD (Institute of Cardiovascular Science, UCL, London); Edward Bove, MD, and Adam Dorfman, MD (University of Michigan, Ann Arbor, Mich); G. Hamilton Baker, MD, and Anthony Hlavacek (Medical University of South Carolina, Charleston, SC); Francesco Migliavacca, PhD, Giancarlo Pennati, PhD, and Gabriele Dubini, PhD (Politecnico di Milano, Milan); Alison Marsden, PhD (University of California, San Diego, San Diego, Calif); Irene Vignon-Clementel (Institut national de recherche en informatique et en automatique, Paris); Richard Figliola, $\mathrm{PhD}$, and John McGregor, $\mathrm{PhD}$ (Clemson University, Clemson, SC).

Received for publication July 14, 2014; revisions received Sept 12, 2014; accepted for publication Oct 4, 2014; available ahead of print Nov 14, 2014.

Address for reprints: Alison L. Marsden, PhD, Mechanical and Aerospace Engineering Department, 9500 Gilman Drive, University of California San Diego, La Jolla, CA 92093-0411 (E-mail: amarsden@ucsd.edu).

$0022-5223 / \$ 36.00$

Copyright $\odot 2015$ by The American Association for Thoracic Surgery http://dx.doi.org/10.1016/j.jtcvs.2014.10.035
For neonates with single-ventricle physiology, including those with hypoplastic left heart syndrome, initial palliation with a systemic-pulmonary shunt, such as a modified Blalock-Taussig (mBT) shunt, is often required before initiating cavopulmonary circulation. ${ }^{1,2}$ However, outcomes after mBT shunt placement remain unsatisfactory, and management of shunt-dependent circulation can be challenging. In addition to the physiologic consequences of obligatory volume load to the single ventricle, and of continuous diastolic runoff of systemic and coronary perfusion, the $\mathrm{mBT}$ shunt has no growth potential and is liable to thrombose, which can lead to profound cyanosis and sudden death. ${ }^{3,4}$ Indeed, first-stage palliation for single-ventricle physiology in which a systemic-to-pulmonary shunt is the sole source of pulmonary blood flow still carries some of the highest morbidity and mortality rates related to congenital heart surgery.

Although a superior cavopulmonary connection, such as a bidirectional Glenn (BDG), would install a more stable source of pulmonary blood flow and mitigate the various physiologic disadvantages of shunt-dependent circulation, experiences with neonatal or early Glenn circulation were unsatisfactory. In an initial report ${ }^{6}$ of outcomes following the classic Glenn procedure in 537 patients, the highest 
mortality was observed when the superior cavopulmonary circulation was established in patients age less than 1 month. The major causes of death were primarily due to inadequate pulmonary blood flow, and in some cases, superior vena cava (SVC) syndrome. Other less-formal attempts to use the early Glenn procedure have led to similar poor outcomes. ${ }^{7-9}$

In the field of fluid mechanics in engineering, the wellknown concept of an "ejector pump" has been widely adopted in industrial applications; it consists of injecting a high-energy flow stream to drive a lower-energy flow stream, elevating downstream pressure while maintaining low upstream pressure. We hypothesize that such a fluidmechanical advantage can be adopted in superior cavopulmonary circulation, in which the low-energy SVC blood flow can be assisted by an additional source of high-energy flow from the systemic circulation. In this novel circulatory arrangement, termed an "assisted bidirectional Glenn" (ABG), a shunt is constructed between the innominate artery and the SVC (in an end-to-side manner) after a BDG anastomosis. A clip is applied to the SVC end of the shunt to produce a Venturi jet effect and augment pulmonary pressure without a significant increase in SVC pressure.

We wanted to assess the conceptual feasibility of the ABG, and quantify the hemodynamic consequences when the pulmonary vascular resistance (PVR) changes as a result of the change from postnatal to infant physiology. We adopted a validated multiscale modeling approach that combines detailed 3-dimensional computational fluid dynamics models with a hydraulic network of the entire cardiopulmonary circulation. Using clinical data and realistic neonatal anatomy, the initial results on the feasibility of using the ABG are reported in this modeling study, including those relating to pulmonary blood flow, ventricular volume load, systemic and mixed venous saturations, systemic and coronary oxygen delivery, and SVC pressure.

\section{METHODS}

\section{Assisted Bidirectional Glenn}

In the $\mathrm{ABG}$ procedure (Figure 1), the SVC is connected to the right pulmonary artery as in the BDG procedure; in addition, a shunt is inserted between the innominate artery and the SVC near the SVC-PA junction in an end-to-side manner. From a fluid-mechanics perspective, flow in the systemic circulation has high "potential" energy because of its high pressure. In the standard $\mathrm{mBT}$ shunt circulation, this energy is dissipated (ie, lost) through the restrictive conduit, and the pressure level is reduced to that of the pulmonary circulation to avoid pulmonary hyperperfusion and hypertension.

With the ABG, however, we attempt to harvest this energy to augment SVC flow, using the ejector-pump concept. To reproduce the Venturi effect of an ejector pump, a nozzle is needed that increases the velocity and decreases the pressure of the high-energy flow, thereby converting potential energy to kinetic energy. In this initial conceptualization of the ABG procedure, this effect is accomplished by applying a ligaclip to the distal shunt, near its junction to the SVC, to acutely limit the shunt diameter and create a Venturi jet directed into the BDG circulation. In an ideal scenario, the kinetic energy of the high-velocity flow is thus converted back into pressure (potential energy) in the diffuser section of the ejector pump, rather than being lost to dissipation. With the ABG, the distal section of the SVC acts partly as a diffuser.

As shown in Figure 2, a high-energy flow from the systemic circulation can be used to assist the lower-energy SVC flow and elevate the pressure at the distal pulmonary artery. This elevated pressure is used to provide higher pulmonary blood flow, without elevating the SVC pressure. Therefore, with the ABG, pulmonary perfusion could be enhanced compared with that with the BDG, while mitigating the disadvantages associated with $\mathrm{mBT}$ shunt circulation.

\section{Multiscale Simulations}

A previously well-described multiscale framework is adopted in this study to couple local hemodynamics to a closed-loop model of the circulatory physiology. ${ }^{10,11}$ In this framework, the computational domain is decomposed into: (1) a 3-dimensional (3D) model of the large vessels for which the Navier-Stokes equations have been solved, and (2) a 0 -dimensional (OD) lumped parameter network (LPN) representing the behavior of the heart and closed-loop circulation (Figure 3). A stable and efficient multidomain approach is used to numerically couple the $3 \mathrm{D}$ and OD domains, as in our prior work. ${ }^{12}$

The 3D, idealized, geometric models were constructed based on a former study of the mBT shunt ${ }^{13}$ (Figure 1). Three models were created using clinically derived geometries and realistic anatomy: a $3.5-\mathrm{mm}$ mBT shunt, a BDG, and an ABG. All models include the aortic arch, 4 upper branches, 2 coronary arteries, and the branch pulmonary arteries. The SVC diameter is $4.5 \mathrm{~mm}$, and the BDG model was created using a simple SVC to right pulmonary artery anastomosis. The shunt between the innominate artery and the SVC in the ABG model is a $2.5-\mathrm{mm}$ tube with a distal clip that reduced the luminal diameter to $1.5 \mathrm{~mm}$. The ABG shunt is about one third the length, and therefore has one third the flow resistance of a standard $\mathrm{mBT}$ shunt, making the shorter, $2.5-\mathrm{mm}$ tube roughly equivalent in resistance to a standard $3.5-\mathrm{mm} \mathrm{mBT}$ shunt.

The adopted LPN is based on prior studies of mBT shunt circulation. ${ }^{13-15}$ Separate blocks model the upper body, lower body, pulmonary bed, coronary circulation, and heart. These blocks are partitioned into arterial-bed, capillary-bed, and venous-bed sections. Component values were obtained from a prior study that incorporated clinical catheterization and angiographic data from 28 Norwood-procedure patients. ${ }^{16}$ The upper-body venous return was directly connected to the SVC in the ABG and BDG LPNs. To avoid regurgitant flow, a coupled Dirichlet boundary was imposed at the ascending aorta. The total excess blood volume in the LPN is preserved temporally to improve cyclic convergence and accuracy. ${ }^{14}$ To mimic the autoregulation mechanism, the total excess blood volume was adjusted among various simulations to keep aortic pressures fixed. To examine the influence of high PVR in the early postnatal period, simulations were performed with a high and a low resistance level (7 and 2.3 Wood units- $\mathrm{m}^{2}$, respectively) in each model. Therefore, a total of 6 simulations were performed.

For the 3D domain, we assumed an incompressible Newtonian fluid and rigid walls. Blood density and viscosity were set to $1060 \mathrm{~kg} / \mathrm{m}^{3}$ and $0.004 \mathrm{~Pa}-\mathrm{s}$, respectively. Simulations were performed using an in-house finite-element solver. The time-step size of the 3D solver was set to $0.5 \mathrm{~ms}$. A specialized preconditioner and an efficient data-structure algorithm were employed in solving the linear system of equations. ${ }^{17,18}$ Due to the presence of backflow in the simulations, special care was taken to avoid rapid simulation divergence at coupled Neumann boundaries by using a minimally intrusive backflow stabilization method. ${ }^{19}$ More details on the numeric methods, model construction process, mesh-convergence study, and oxygen-delivery calculations are available elsewhere. ${ }^{13}$

\section{RESULTS}

The results of the 6 simulations are shown in Table 1 and Figure 4. Simulations of the ABG procedure without the 

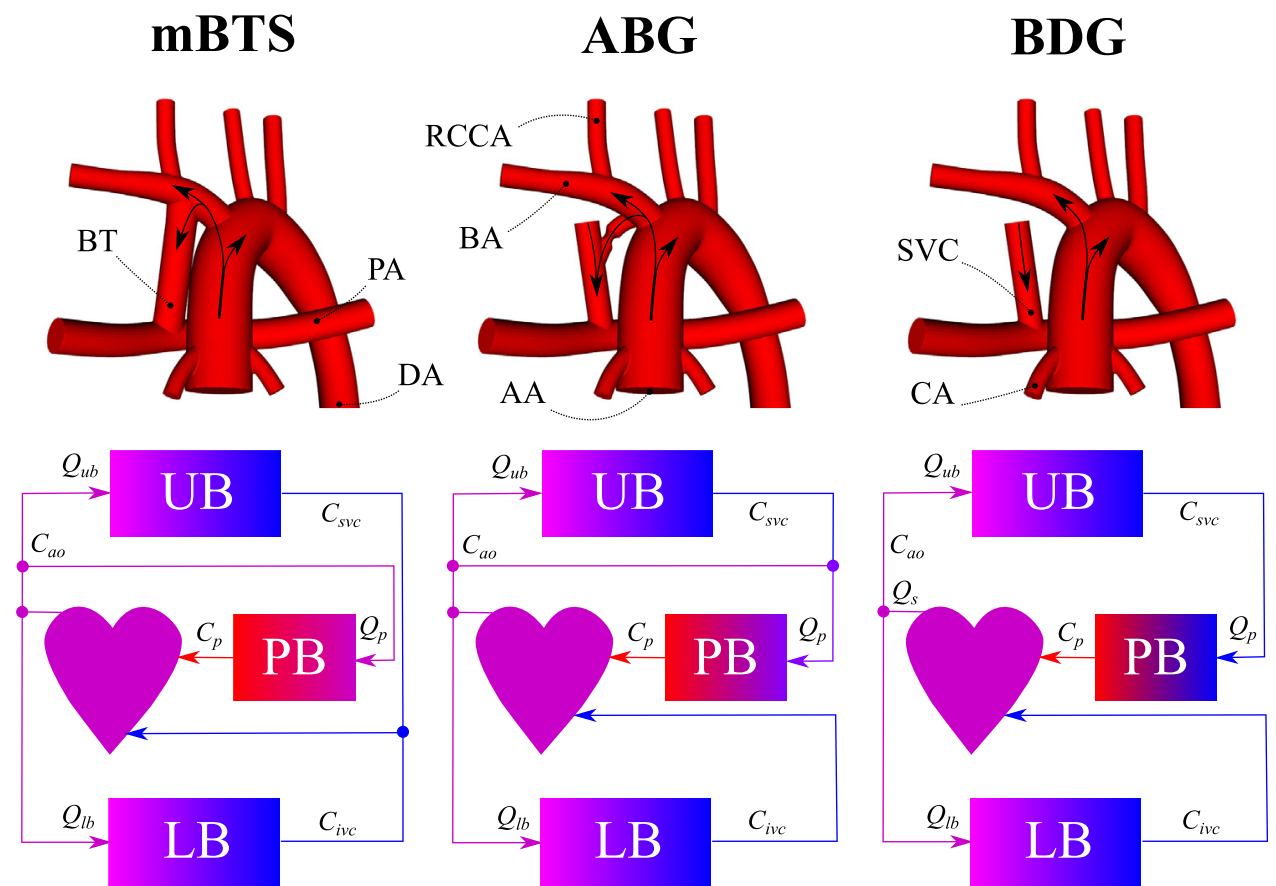

FIGURE 1. Idealized models of the mBTS, ABG, and BDG (top row). Colors in the circulation schematics (bottom row) represent the level of oxygen concentration. $m B T S$, Modified Blalock-Taussig shunt; $A B G$, assisted bidirectional Glenn; $B D G$, bidirectional Glenn; $R C C A$, right common carotid artery; $B T$, Blalock-Taussig shunt; $B A$, brachiocephalic artery; $S V C$, superior vena cava; $P A$, pulmonary arteries; $D A$, descending aorta; $A A$, ascending aorta; $C A$, coronary arteries; $Q_{u b}$, upper-body flow rate; $U B$, upper body; $C_{s v c}$, superior vena cava oxygen concentration; $C_{a o}$, aortic oxygen concentration; $Q_{s}$, systemic flow rate; $C_{p}$, pulmonary vein oxygen concentration; $P B$, pulmonary bed; $Q_{p}$, pulmonary flow rate; $Q_{l b}$, lower-body flow rate; $L B$, lower body; $C_{i v c}$, inferior vena cava oxygen concentration.

distal clipping were also performed, although the results were unsatisfactory. Without the clipping, at low PVR, SVC pressure increased to $12.3 \mathrm{~mm} \mathrm{Hg}$; oxygen delivery decreased slightly to $3.32 \mathrm{~mL}_{\mathrm{O} 2} / \mathrm{s}$; and pulmonary flow increased to $1.01 \mathrm{~L} / \mathrm{min}$, resulting in pulmonary overcirculation. This result demonstrates the importance of clipping the shunt at its distal connection to the SVC, to restrict flow and reproduce the ejector-pump effect. With the clipped shunt, pressure upstream from the SVC-shunt anastomosis is lower than the pressure downstream. This finding provides evidence that the energy of the systemic flow is partly transferred to the SVC flow and that the ejectorpump concept is feasible, although perhaps not optimal, with use of the clip. Therefore, to avoid confusion, only results from the $\mathrm{ABG}$ configuration in which distal clipping was used are reported, along with results from the $\mathrm{mBT}$ shunt and BDG models.

As expected, compared to the $\mathrm{mBT}$ shunt model, the BDG offloads the ventricle and lowers cardiac output while maintaining oxygen delivery with higher SVC pressure. However,

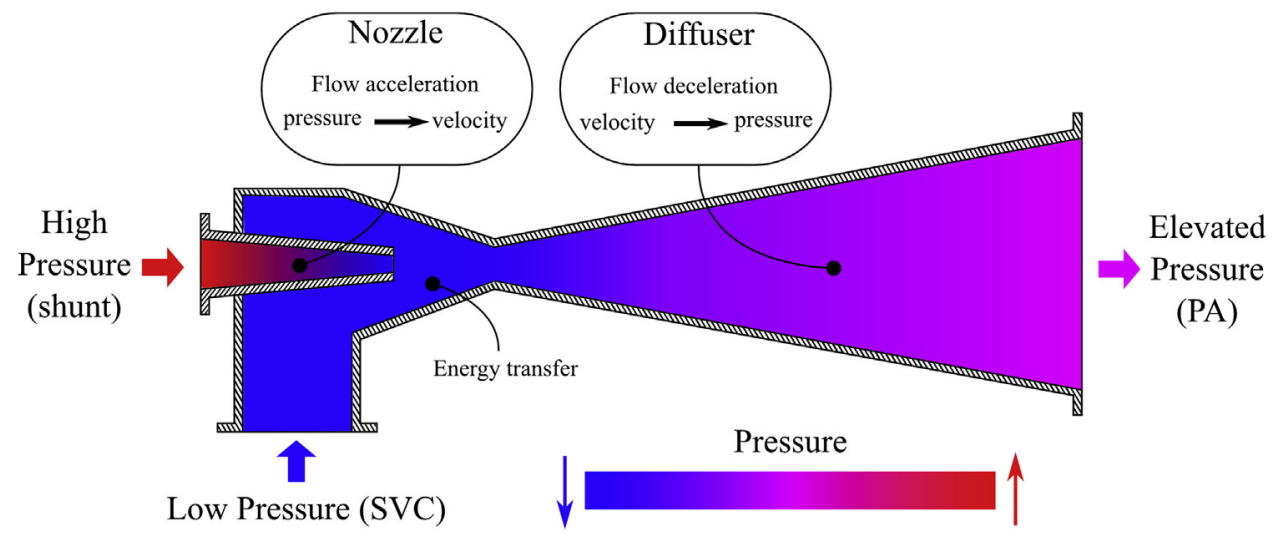

FIGURE 2. A schematic of an industrial ejector pump. This device transfers the energy of higher-pressure flow to lower-pressure flow, thus elevating the pressure at the outlet. Based on the same concept, flow through the SVC can be assisted by flow through the shunt to obtain a higher pressure at the PA without increasing SVC pressure. PA, Pulmonary artery; SVC, superior vena cava. 


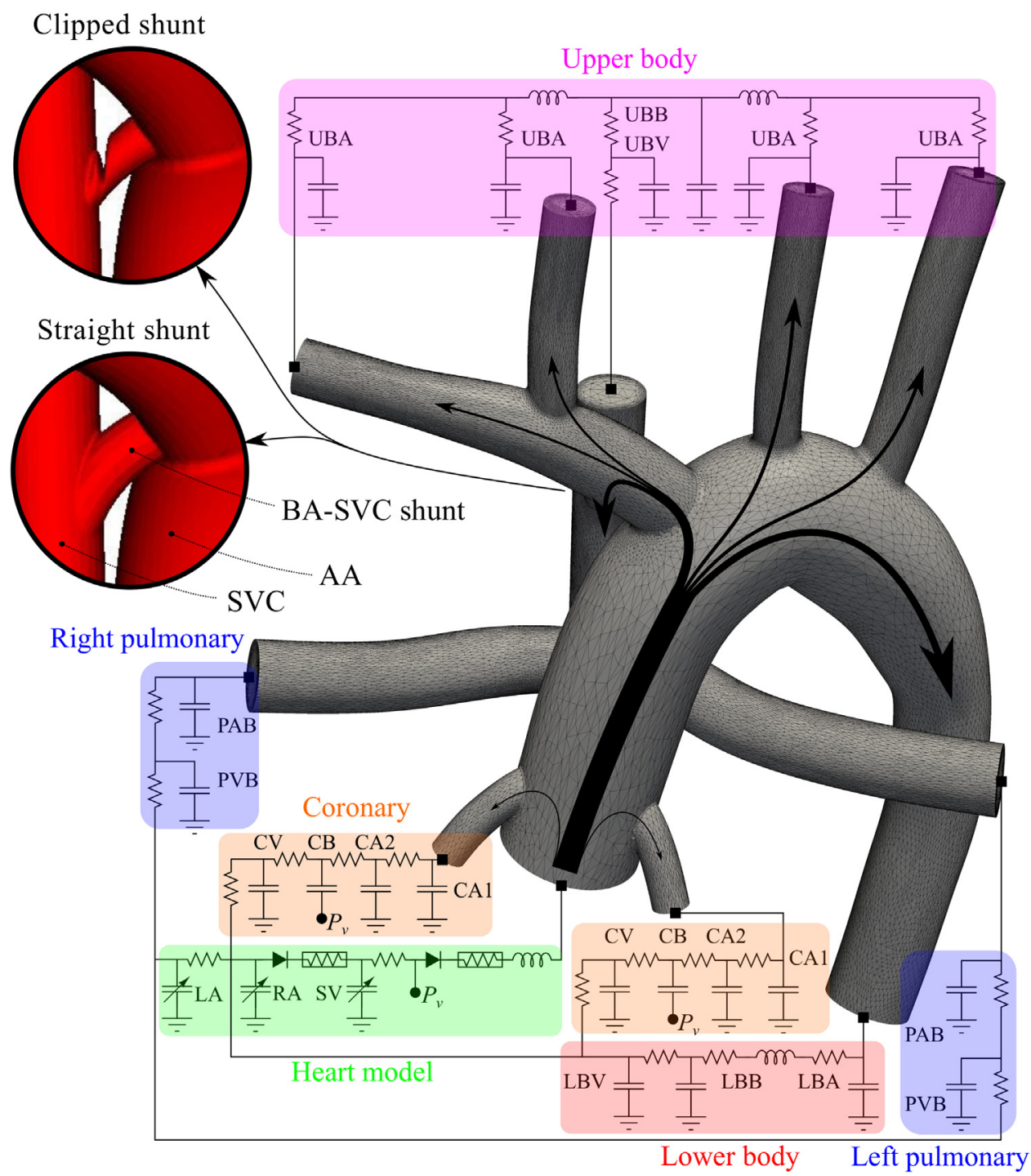

FIGURE 3. Lumped parameter network coupled to the assisted bidirectional anatomy. This network contains blocks for the UBA, UBB, UBV, PAB, PVB, LBA, LBB, LBV, 2 CAs, the CB, CV, LA, RA, and SV. Two geometries, clipped and straight, are shown for the BA-SVC shunt. In the assisted bidirectional Glenn, pulmonary flow is supplied through the SVC and the shunt. $U B B$, Upper-body capillary bed; $U B A$, upper-body arteries; $U B V$, upper-body veins; $B A-S V C$, brachiocephalic-superior vena cava; $A A$, ascending aorta; $S V C$, superior vena cava; $P A B$, pulmonary artery bed; $P V B$, pulmonary vein bed; $C V$, coronary veins; $C B$, coronary capillary bed; $C A$, coronary artery; $P_{v}$, ventricular pressure; $L A$, left atrium; $R A$, right atrium; $S V$, single ventricle; $L B V$, lower-body veins; $L B B$, lower-body capillary bed; $L B A$, lower-body arteries.

both cardiac output and ventricular load are comparable in the ABG and BDG models (Figure 4). Although pulmonary blood flow is highest in the mBT shunt model, the ABG improved pulmonary blood flow compared with the BDG, and the ABG model achieves the highest oxygen saturation and oxygen delivery regardless of PVR. At a PVR of 7 Wood units- $\mathrm{m}^{2}$, the ABG model predicted an SVC pressure of $15 \mathrm{~mm} \mathrm{Hg}$, which decreases to $8 \mathrm{~mm} \mathrm{Hg}$ at a PVR of 2.3 Wood units- $\mathrm{m}^{2}$. The flow rate through the clipped shunt in the ABG model can be found from Table 1, by subtracting $\mathrm{Q}_{\mathrm{ub}}$ from $\mathrm{Q}_{\mathrm{p}}\left(\mathrm{Q}_{\mathrm{p}}-\mathrm{Q}_{\mathrm{ub}}\right)$, producing a value of $0.22 \mathrm{~L} / \mathrm{min}$ at low PVR, approximately one third of the pulmonary blood flow. Although the clipping area may vary in surgical implementation, we would expect a linear relationship between the clipping area and shunt flow rate, according to Bernoulli's equation, so that changes in hemodynamics with clipping area may be interpolated from the ABG and BDG results. As all simulations were deterministic, and only a single geometry of each type was tested, there are no statistics to report based on Table 1 data.

\section{DISCUSSION}

This computational simulation study evaluated the concept of augmentation of pulmonary blood flow in a 
TABLE 1. Simulation results of various surgical options at normal and high PVR $\left(\mathrm{N}=2.3, \mathrm{H}=7\right.$ Wood units- $\left.{ }^{2}\right)$

\begin{tabular}{lccccccc}
\hline & & mBTS & BDG & ABG & MBTS & BDG & ABG \\
Unit & PVR & $\mathbf{N}$ & $\mathbf{N}$ & $\mathbf{N}$ & $\mathbf{H}$ & $\mathbf{H}$ & $\mathbf{H}$ \\
\hline $\mathrm{OD}$ & $\mathrm{mL}_{\mathrm{O} 2} / \mathrm{s}$ & 2.96 & 3.25 & 3.38 & 2.92 & 2.79 & 3.07 \\
$\mathrm{OD}_{\text {cor }}$ & $\mathrm{mL}_{\mathrm{O} 2} / \mathrm{s}$ & 0.22 & 0.24 & 0.26 & 0.22 & 0.22 & 0.25 \\
$\mathrm{OD}_{\text {ub }}$ & $\mathrm{mL}_{\mathrm{O} 2} / \mathrm{s}$ & 1.30 & 1.39 & 1.41 & 1.28 & 1.11 & 1.16 \\
$\mathrm{HL}$ & $\mathrm{Nm} / \mathrm{min}$ & 28.0 & 12.5 & 15.0 & 26.7 & 10.5 & 13.6 \\
$\mathrm{CO}$ & $\mathrm{L} / \mathrm{min}$ & 2.01 & 1.17 & 1.31 & 1.93 & 1.06 & 1.23 \\
$\mathrm{Q}_{\mathrm{p}} / \mathrm{Q}_{\mathrm{s}}$ & - & 0.85 & 0.43 & 0.58 & 0.71 & 0.40 & 0.54 \\
$\mathrm{Q}_{\mathrm{s}}$ & $\mathrm{L} / \mathrm{min}$ & 1.09 & 1.17 & 1.13 & 1.13 & 1.06 & 1.06 \\
$\mathrm{Q}_{\mathrm{p}}$ & $\mathrm{L} / \mathrm{min}$ & 0.92 & 0.50 & 0.65 & 0.80 & 0.42 & 0.57 \\
$\mathrm{Q}_{\mathrm{lb}}$ & $\mathrm{L} / \mathrm{min}$ & 0.61 & 0.67 & 0.66 & 0.63 & 0.64 & 0.66 \\
$\mathrm{Q}_{\mathrm{ub}}$ & $\mathrm{L} / \mathrm{min}$ & 0.48 & 0.50 & 0.47 & 0.50 & 0.42 & 0.40 \\
$\mathrm{Q}_{\mathrm{cor}}$ & $\mathrm{mL} / \mathrm{min}$ & 8.16 & 8.71 & 8.65 & 8.42 & 8.29 & 8.64 \\
$\mathrm{P}_{\mathrm{ao}}$ & $\mathrm{mm} \mathrm{Hg}$ & 52.1 & 54.6 & 55.2 & 53.7 & 51.8 & 54.9 \\
$\mathrm{P}_{\mathrm{SVC}}$ & $\mathrm{mm} \mathrm{Hg}$ & 2.5 & 6.0 & 7.9 & 2.4 & 11.1 & 14.9 \\
$\mathrm{P}_{\mathrm{PA}}$ & $\mathrm{mm} \mathrm{Hg}$ & 8.1 & 4.7 & 6.2 & 17.9 & 10.1 & 13.5 \\
Sat $_{\text {ao }}$ & $\%$ & 72.7 & 74.5 & 80.1 & 68.9 & 70.3 & 77.4 \\
Sat $_{\mathrm{SVC}}$ & $\%$ & 48.2 & 51.1 & 55.2 & 45.5 & 42.5 & 48.2 \\
Sat $_{\text {IVC }}$ & $\%$ & 53.7 & 57.1 & 62.4 & 50.5 & 52.0 & 59.8 \\
\hline
\end{tabular}

$m B T S$, Modified Blalock-Taussig shunt; $B D G$, bidirectional Glenn; $A B G$, assisted bidirectional Glenn; $P V R$, pulmonary vascular resistance; $N$, normal; $H$, high; $O D$, systemic oxygen delivery; $O D_{c o r}$, coronary oxygen delivery; $O D_{u b}$, upperbody oxygen delivery; $H L$, approximate load on the single ventricle; $C O$, ascending aorta (cardiac output) average flow rate; $Q_{p}$, pulmonary average flow rate; $Q_{s}$, systemic average flow rate; $Q_{l b}$, lower-body average flow rate; $Q_{u b}$, upper-body average flow rate; $Q_{c o r}$, coronary average flow rate; $P_{a o}$, aortic average pressure; $P_{S V C}$, superior vena cava average pressure; $P_{P A}$, pulmonary artery average pressure; $S a t_{a o}$, aortic percentage oxygen saturation; $S_{S t_{S V C}}$, superior vena caval percentage oxygen saturation; Sat $_{I V C}$, inferior vena caval percentage oxygen saturation.

superior cavopulmonary connection using an ABG shunt from the systemic circulation to act as an ejector pump. The model seems to achieve optimal systemic and mixed venous saturation, systemic and coronary oxygen delivery, and acceptable SVC pressures, even when PVR is high. Although preliminary, the potential added benefits of the $\mathrm{ABG}$ as a first-stage palliation compared with the mBT shunt could be numerous. By eliminating the mBT shunt as an initial palliation, the typical 3-stage surgical palliation for the single-ventricle pathway could be reduced to 2 stages. With the superior cavopulmonary connection as the primary source of pulmonary blood flow, a more reliable and stable circulation could be achieved with reduced risk of thrombosis and cyanosis. Although thrombotic risk remains poorly understood, even if the systemic-to-SVC ejector pump undergoes acute occlusion, sudden death or profound cyanosis could potentially be avoided, as the circulation would simply revert to that with a standard BDG, although aggressive anticoagulation measures might be needed to avoid this conversion in the early neonatal period.

Moreover, the ABG model has important physiologic advantages. The combination of having the superior venous return directed to the pulmonary circulation and limiting the runoff through the systemic-SVC shunt allows early volume unloading of the single ventricle and the associated improvement in mechanical efficiency. The elimination of deoxygenated blood from the upper body in atrial mixing with pulmonary venous return can lead to higher systemic saturation compared with shunt-dependent circulation, as observed in our model. The removal of diastolic runoff improves both cerebral and coronary perfusion and oxygen delivery. In addition, operations have been performed, similar to the $\mathrm{ABG}$ procedure, in which a BDG is supplemented with a shunt from the systemic circulation to either the SVC or the pulmonary artery (P. del Nido, MD, personal communication, July 2014). Therefore, an end-to-side anastomosis of a shunt to the SVC appears to be technically feasible.

Adopting a systemically derived accessory pulmonary flow in the BDG circulation has been reported previously both clinically and investigationally, ${ }^{20-23}$ although typically not during the neonatal period. The reported mortality and morbidity rates in patients with accessory flow differ among institutions. Van deWal and colleagues ${ }^{20}$ reported no influence on the survival rate in a study of 205 patients with an average age of 5.6 years; McElhinney and colleagues ${ }^{21}$ reported higher, yet statistically insignificant, mortality in a study of 160 patients with an average age of 7.8 months; and Mainwaring and colleagues ${ }^{22}$ reported higher mortality in study of
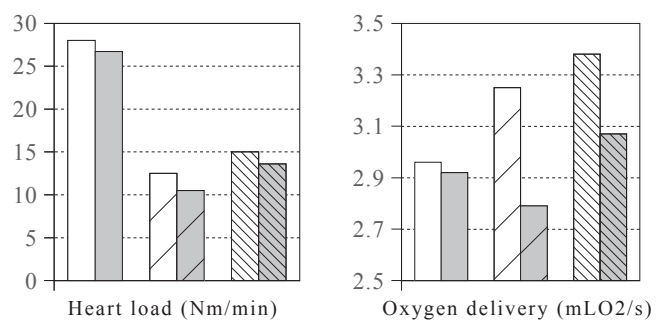

$\square \mathrm{mBTS} / \mathrm{N}$

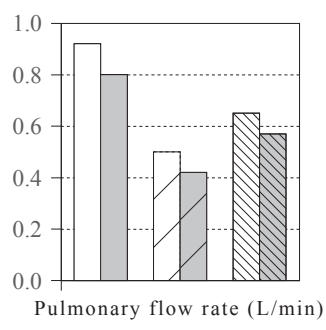

$P \mathrm{BDG} / \mathrm{N} P \mathrm{BDG} / \mathrm{H}$

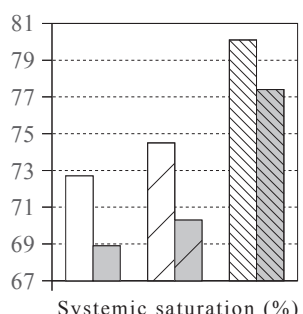

Systemic saturation (\%)

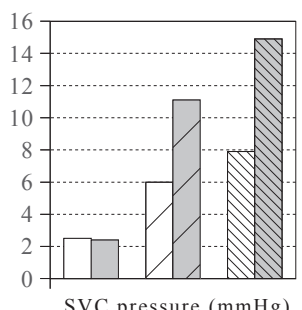

SVC pressure $(\mathrm{mmHg})$

FIGURE 4. mBTS, BDG, and ABG heart load, oxygen delivery, pulmonary flow rate, systemic oxygen saturation, and SVC pressure at normal (white) and high (gray) PVR. SVC, Superior vena cava; $m B T S$, modified Blalock-Taussig shunt; $N$, normal; $H$, high; $B D G$, bidirectional Glenn; $A B G$, assisted bidirectional Glenn. 
149 patients with an average age of 10 months. ${ }^{23}$ Earlier completion of Fontan was reported in patients with accessory flow, and it has been argued that if hepatic-factor is important for the prevention of pulmonary arteriovenous malformation, then having accessory pulsatile flow might represent an advantage.$^{20}$ In addition, it has been suggested that accessory flow may improve exercise tolerance by providing a variable pulmonary flow. $^{24}$

Although these prior studies do not provide evidence of clinical applicability in a neonate, they do at least partially support the technical feasibility of the ABG procedure. Consistent with the present study results, prior studies that did not restrict the accessory shunt showed unacceptably high SVC pressure and ventricular load, with poor clinical consequences, despite the advantages of higher oxygen saturation levels and higher pulmonary flow rate. Thus, clipping the shunt in the ABG not only creates the Venturi jet effect that is essential to recapitulate the ejector-pump mechanism, but also restricts the shunt flow to reduce SVC pressure and ventricular load.

Early application of the BDG as a first-stage palliation has been traditionally avoided, based on poor outcomes from Glenn and others. However, the ABG offers a distinctly different strategy and perspective that make it worthy of continued investigation. First, early Glenn experiences were derived from the classic Glenn with unilateral right pulmonary artery anastomosis, and therefore, single-lung runoff to the SVC. SVC pressures with runoff from 2 lungs will likely result in lower SVC pressures. Second, the majority of deaths after early Glenn anastomosis were due to inadequate pulmonary blood flow, cyanosis, insufficient oxygen delivery, and associated respiratory complications. Based on these simulation results, avoidance of these complications seems to be the primary advantage of the ABG circulation.

Nonetheless, the main impediment to clinical application of the $\mathrm{ABG}$ as a first-stage palliation remains the potential for high SVC pressures. Although SVC pressure at the highest simulated PVR, corresponding to the neonatal period, is $15 \mathrm{~mm} \mathrm{Hg}$, the high SVC pressure remains a valid concern. The level of SVC pressure that would be clinically unacceptable in terms of upper-body edema and adverse effects on cerebral perfusion has not been determined. But, it is clear that SVC pressures near $20 \mathrm{~mm} \mathrm{Hg}$ would be poorly tolerated in the neonatal period. To mitigate the SVC pressure increase in the ABG, we used a high-velocity jet flow achieved by simple clipping of the shunt at its anastomosis with the SVC. This configuration is technically easy from a surgical perspective, but it may not be adequately optimized in terms of fluid mechanics. For maximal energy recovery, it is essential to reduce viscous dissipation by using a minimal-length shunt, a diffuser-shaped SVC, and a proper anastomosis angle and cross-section. Clearly, a wide range of shunt/jet designs should be considered, to minimize SVC pressure and decrease the potential for SVC syndrome.

Early thrombosis of the ejector-pump shunt in the ABG is a potential hazard. Although a complete loss of pulmonary blood flow can be avoided in the ABG, as opposed to the scenario of shunt occlusion in a shunt-dependent single-ventricle circulation, there remains a risk of early "conversion" to the BDG by shunt occlusion. One potential mitigating factor is that, because the ABG shunt is so restrictive, flow velocity through the shunt is very high. Moreover, as the shunt segment is about one third the length of a standard BT shunt, there will be lower flow residence time, which is a common cause of thrombosis. Nonetheless, no mathematical simulation can recreate all the potential biological responses, including the thrombosis/coagulation cascade. Therefore, we are actively working on an animal model to examine issues such as ABG shunt thrombosis in an in vivo setting.

An important corollary of this study is that it highlights the potential of multiscale computational modeling as a platform for initial testing and feasibility studies of novel surgical concepts that would otherwise be ethically or technically infeasible to perform clinically. Although many operative innovations that have revolutionized surgical care of congenital heart defects have resulted directly from application in operating theaters, this practice is no longer socially or medicolegally acceptable. Therefore, a modeling scheme that can reproduce realistic physiologic and hemodynamic responses to novel surgical reconstructions or concepts is an important paradigm that can provide a needed avenue to encourage new ideas and innovations.

We are actively expanding modeling investigations of the ABG concept to include patient-specific models under more-realistic physiologic and geometric conditions. Optimization and uncertainty quantification tools will allow for refinement of the shunt/jet design and accuracy assessment. A more extensive LPN may be needed to accurately capture the physiologic response of the circulatory system to the large variations imposed by drastically different surgeries. We have constructed a mock circulatory bed to physically test the ABG concept in simulated neonatal circulation. Based on these results, in vitro animal and clinical studies can be designed and proposed. One potential clinical use of early ABG installation is for patients with a failing BDG circulation owing to inadequate pulmonary flow; the $\mathrm{ABG}$ would be an alternative to take-down of the BDG to restore a shunt circulation.

\section{Limitations}

Limitations of simulation methods in this study include the use of rigid walls ${ }^{25}$; however, prior Fontan and $\mathrm{mBT}$ shunt simulations have shown limited effects of fluid 
structure interaction on pressures and flow distribution results. Additional limitations include a Newtonian flow assumption, limited models for oxygen uptake and saturation, and a simplified model of the autoregulatory mechanism. ${ }^{14}$ Future studies should incorporate additional clinical data to study a wider range of possible physiologic conditions that may occur during the neonatal period, and multiple patient-specific models, so that statistics can be gathered. A further limitation of the model is that it did not incorporate a growth model for scaling between the stage 1 and stage 2 surgeries, owing to a lack of clinical data to support model development, although 2 levels of PVR were examined, to account for the drop in PVR during the neonatal period. In addition, the model did not account for the biochemistry of thrombus formation.

\section{CONCLUSIONS}

The reasons for high mortality rates among patients who undergo surgery for cavopulmonary connection in the neonatal period are not fully understood. If low pulmonary perfusion and low oxygenation are the leading causes, the ABG could have potential as a viable alternative to the mBT shunt, as it may reduce the heart load and cardiac output significantly, provide higher levels of oxygenation, and add redundancy to sources of pulmonary blood flow. In addition, it may reduce the risk of mortality caused by blockage of the mBT shunt, and potentially reduce the total number of palliative surgeries needed to 2. High SVC pressure remains a concern with the $\mathrm{ABG}$ approach, and future studies are required to address this potential drawback.

\section{References}

1. Norwood W, Kirklin J, Sanders S. Hypoplastic left heart syndrome: experience with palliative surgery. Am J Cardiol. 1980;45:87-91.

2. Norwood W, Lang P, Castaneda A, Campbell D. Experience with operations for hypoplastic left heart syndrome. J Thorac Cardiovasc Surg. 1981;82:511-9.

3. Wong R, Baum V, Sangwan S. Truncus arteriosus: recognition and therapy of intra-operative cardiac ischemia. Anesthesiology. 1991;74:378-80.

4. Bartram U, Grnenfelder J, Praagh RV. Causes of death after the modified Norwood procedure: a study of 122 postmortem cases. Ann Thorac Surg. 1997; 64:1795-802.

5. Tamisier D, Vouhe P, Vernant F, Leca F, Massot C, Neveux J. Modified Blalock-Taussig shunts: results in infants less than 3 months of age. Ann Thorac Surg. 1990;49:797-801.

6. Glenn WW, Browne M, Whittemore R. Circulatory bypass of the right side of the heart: cava-pulmonary artery shunt-indications and results (report of a collected series of 537 cases). In: Cassels DE, ed. The Heart and Circulation in the Newborn and Infant. New York: Grune \& Stratton; 1966:345-57.
7. Glenn WW. Circulatory bypass of the right side of the heart. IV. Shunt between superior vena cava and distal right pulmonary artery; report of clinical application. N Engl J Med. 1958;259:117-20.

8. Glenn WW, Patino JF. Circulatory by-pass of the right heart. I. Preliminary observations on the direct delivery of vena caval blood into the pulmonary arterial circulation. Azygos vein-pulmonary artery shunt. Yale J Biol Med. 1954;27:147-51

9. Nuland SB, Glenn WW, Guilfoil P. Circulatory bypass of the right heart. III. Some observations on long-term survivors. Surgery. 1958;43:184.

10. Hsia T-Y, Cosentino D, Corsini C, Pennati G, Dubini G, Migliavacca F. Use of mathematical modeling to compare and predict hemodynamic effects between hybrid and surgical Norwood palliations for hypoplastic left heart syndrome. Circulation. 2011;124:S204-10.

11. Bove E, Migliavacca F, de Leval M, Balossino R, Pennati G, Lloyd T, et al. Use of mathematic modeling to compare and predict hemodynamic effects of the modified Blalock-Taussig and right ventricle-pulmonary artery shunts for hypoplastic left heart syndrome. J Thorac Cardiovasc Surg. 2008;136:312-20.

12. Esmaily-Moghadam M, Vignon-Clementel I, Figliola R, Marsden A. A modular numerical method for implicit 0D/3D coupling in cardiovascular finite element simulations. J Comput Phys. 2013;224:63-79.

13. Esmaily-Moghadam M, Migliavacca F, Vignon-Clementel I, Hsia T, Marsden A. Optimization of shunt placement for the Norwood surgery using multi-domain modeling. J Biomech Eng. 2012;134:051002.

14. Esmaily-Moghadam M, Murtuza B, Hsia T, Marsden A. Simulations reveal adverse hemodynamics in patients with multiple systemic to pulmonary shunts. J Biomech Eng. 2014 [in press].

15. Lagana K, Balossino R, Migliavacca F, Pennati G, Bove E, de Leval M, et al Multiscale modeling of the cardiovascular system: application to the study of pulmonary and coronary perfusions in the univentricular circulation. $J$ Biomech 2005;38:1129-41.

16. Migliavacca F, Pennati G, Dubini G, Fumero R, Pietrabissa R, Urcelay G, et al Modeling of the Norwood circulation: effects of shunt size, vascular resistances, and heart rate. Am J Physiol Heart Circ Physiol. 2001;280:H2076-86.

17. Esmaily-Moghadam M, Bazilevs Y, Marsden AL. A new preconditioning technique for implicitly coupled multidomain simulations with applications to hemodynamics. Comput Mech. 2013;52:1141-52.

18. Esmaily-Moghadam M, Bazilevs Y, Marsden A. Impact of data distribution on the parallel performance of iterative linear solvers with emphasis on CFD of incompressible flows. Comput Mech. October 25, 2014 [Epub ahead of print].

19. Esmaily-Moghadam M, Bazilevs Y, Hsia T, Vignon-Clementel I, Marsden A. A comparison of outlet boundary treatments for prevention of backflow divergence with relevance to blood flow simulations. Comput Mech. 2011;48:277-91.

20. van deWal HJ, Ouknine R, Tamisier D, Levy M, Vouhe PR, Leca F. Bi-directional cavopulmonary shunt: Is accessory pulsatile flow, good or bad? Eur $J$ Cardiothorac Surg. 1999;16:104-10.

21. McElhinney DB, Marianeschi SM, Reddy VM. Additional pulmonary blood flow with the bidirectional Glenn anastomosis: Does it make a difference? Ann Thorac Surg. 1998;66:668-72.

22. Mainwaring RD, Lamberti JJ, Uzark K, Spicer RL, Cocalis MW, Moore JW Effect of accessory pulmonary blood flow on survival after the bidirectional Glenn procedure. Circulation. 1999;100(Suppl 2):II151.

23. Gervaso F, Kull S, Pennati G, Migliavacca F, Dubini G, Luisi VS. The effect of the position of an additional systemic-to-pulmonary shunt on the fluid dynamics of the bidirectional cavo-pulmonary anastomosis. Cardiol Young. 2004;14:38-43.

24. Hunt D, Edwards WS, Deverall PB, Bargeron L. Superior vena cava to right pulmonary artery anastomosis results in 46 infants and children. Thorax. 1970; 25:550-5.

25. Long C, Hsu M-C, Bazilevs Y, Feinstein J, Marsden A. Fluid-structure interaction simulations of the Fontan procedure using variable wall properties. Int J Numer Method Biomed Eng. 2012;28:513-27. 